\title{
A Precursor of Chiral Symmetry Restoration in the Nuclear Medium
}

\author{
T. Hatsuda ${ }^{(1)}$, T. Kunihiro(2), H. Shimizu ${ }^{(3)}$ \\ (1) Physics Department, Kyoto University, Kyoto 606-8502, Japan \\ (2) Faculty of Science and Technology, Ryukoku University, Seta, Otsu-city, 520-2194, Japan \\ (3) Physics Department, Yamagata University, Yamagata 990-8560, Japan
}

(March 6, 2018)

Spectral enhancement near the $2 m_{\pi}$ threshold in the $I=J=0$ channel in nuclei is shown to be a distinct signal of the partial restoration of chiral symmetry. The relevance of this phenomenon with the possible detection of $2 \pi^{0}$ and $2 \gamma$ in hadron-nucleus and photo-nucleus reactions is discussed.

24.85.+p, 14.40.Cs

One of the intriguing phenomena in the physics of strong interactions is the dynamical breaking of chiral symmetry $(\mathrm{DB} \chi)$. This explains the existence of the pion and also dictates most of the low energy phenomena in hadron physics. In the context of the Quantum Chromo Dynamics (QCD), the fundamental theory of strong interactions, $\mathrm{DB} \chi$ is associated with the condensation of quark - anti-quark pairs in the vacuum. This is analogous to the condensation of Cooper pairs in the theory of superconductivity [1]. Furthermore, as the baryon density and/or the temperature are raised, the $\mathrm{QCD}$ vacuum is supposed to undergo a phase transition to the chirally symmetric phase [2]. In particular, some theoretical analyses suggest that a partial restoration of chiral symmetry occurs even at a relatively low baryon density $(\rho)$ close to the nuclear matter density $\rho_{0}=0.17 \mathrm{fm}^{-3}$ [2]. Then, it is of fundamental importance to clarify what observables reflect most properly this partial symmetry restoration. The purpose of this Letter is to give a candidate of such observables and to propose experiments to test the idea with nuclear targets.

The general wisdom of many-body physics [ 3 - 5 tells us that the fluctuation of the order parameter becomes large as the system approaches the critical point of the phase transition. In QCD, this corresponds to a softening of a collective excitation having the same quantum number as that of the vacuum, namely the scalar-isoscalar $(I=J=0)$ meson, $\sigma$ [6]. The softening (the red-shift of the spectrum) in turn causes the decrease of the decay width of $\sigma$ by the phase-space suppression of the reaction $\sigma \rightarrow 2 \pi$. This leads to a conjecture that $\sigma$ may appear as a sharp resonance at finite temperature $(T)$ [6, 6], although it can be elusive due to the large width in the free space [8,9]. A later analysis at $T \neq 0$ showed that the spectral function in the $\sigma$-channel has a characteristic enhancement just above the two-pion threshold [10].

In this Letter, we demonstrate, using a toy model, that the spectral enhancement near the threshold is a distinct signal of the partial chiral restoration also at $\rho \neq 0$. As possible experiments to observe this enhancement, we will propose to detect the neutral-dipion $\left(2 \pi^{0}\right)$ and diphoton $(2 \gamma)$ in reactions with heavy nuclear targets [11. We will also mention the relevance of the softening to the recent data on the near-threshold $\pi^{+} \pi^{-}$production in $\pi^{+}$-nucleus reactions by the CHAOS collaboration [14].

Before presenting our explicit model-calculation, let us describe the general features of the spectral enhancement near the two-pion threshold. Consider the propagator of the $\sigma$-meson at rest in the medium : $D_{\sigma}^{-1}(\omega)=\omega^{2}-m_{\sigma}^{2}$ $-\Sigma_{\sigma}(\omega ; \rho)$, where $m_{\sigma}$ is the mass of $\sigma$ in the tree-level, and $\Sigma_{\sigma}(\omega ; \rho)$ is the loop corrections in the vacuum as well as in the medium. The corresponding spectral function $\rho_{\sigma}(\omega)=-\pi^{-1} \operatorname{Im} D_{\sigma}(\omega)$ reads

$$
\rho_{\sigma}(\omega)=-\frac{1}{\pi} \frac{\operatorname{Im} \Sigma_{\sigma}}{\left(\omega^{2}-m_{\sigma}^{2}-\operatorname{Re} \Sigma_{\sigma}\right)^{2}+\left(\operatorname{Im} \Sigma_{\sigma}\right)^{2}} .
$$

Near the two-pion threshold, the phase space factor gives $\operatorname{Im} \Sigma_{\sigma} \propto \theta\left(\omega-2 m_{\pi}\right) \sqrt{1-\frac{4 m_{\pi}^{2}}{\omega^{2}}}$ in the one-loop order. On the other hand, the partial restoration of chiral symmetry indicates that $m_{\sigma}^{*}$ (the "effective mass" of $\sigma$ defined through $\left.\operatorname{Re} D_{\sigma}^{-1}\left(\omega=m_{\sigma}^{*}\right)=0\right)$ approaches to $m_{\pi}$. Therefore, there exists a density $\rho_{c}$ at which $\operatorname{Re} D_{\sigma}^{-1}\left(\omega=2 m_{\pi}\right)$ vanishes even before the complete $\sigma$ $\pi$ degeneracy takes place; namely $\operatorname{Re} D_{\sigma}^{-1}\left(\omega=2 m_{\pi}\right)=$ $\left[\omega^{2}-m_{\sigma}^{2}-\operatorname{Re} \Sigma_{\sigma}\right]_{\omega=2 m_{\pi}}=0$. At this point, the spectral function is solely dictated by the imaginary part of the self-energy;

$$
\rho_{\sigma}\left(\omega \simeq 2 m_{\pi}\right)=-\frac{1}{\pi \operatorname{Im} \Sigma_{\sigma}} \propto \frac{\theta\left(\omega-2 m_{\pi}\right)}{\sqrt{1-\frac{4 m_{\pi}^{2}}{\omega^{2}}}} .
$$

This shows that, even if there is no sharp resonance in the scalar channel in the free space, there arises a mild (integrable) singularity just above the threshold in the medium. We emphasize that this is a general phenomenon correlated with the partial restoration of chiral symmetry.

To make the argument more quantitative, let us evaluate $\rho_{\sigma}(\omega)$ in a toy model, namely the $\mathrm{SU}(2)$ linear $\sigma$ model:

$$
\begin{aligned}
\mathcal{L}=\frac{1}{4} \operatorname{tr}\left[\partial M \partial M^{\dagger}\right. & -\mu^{2} M M^{\dagger} \\
& \left.-\frac{2 \lambda}{4 !}\left(M M^{\dagger}\right)^{2}-h\left(M+M^{\dagger}\right)\right],
\end{aligned}
$$


where $\operatorname{tr}$ is for the flavor index and $M=\sigma+i \vec{\tau} \cdot \vec{\pi}$. Although the model is not a precise low energy representation of QCD [15], it is known to describe the pion dynamics qualitatively well up to $1 \mathrm{GeV}$ as shown by Chan and Haymaker [16].

The coupling constants $\mu^{2}, \lambda$ and $h$ have been determined in the vacuum to reproduce $f_{\pi}=93 \mathrm{MeV}$, $m_{\pi}=140 \mathrm{MeV}$ as well as the s-wave $\pi-\pi$ scattering phase shift in the one-loop order. Resultant parameters in the $\overline{M S}$ renormalization scheme are given in [10] and are recapitulated for two characteristic cases in TABLE I in which $m_{\sigma}^{\text {peak }}$ is defined as a peak position of $\rho_{\sigma}(\omega)$.

The interaction Lagrangian of $M$ with the nucleon field $N$ with $\mathrm{SU}(2)$ chiral symmetry is modeled as

$$
\mathcal{L}_{I}(N, M)=-g \chi \bar{N} U_{5} N-m_{0} \bar{N} U_{5} N
$$

where we have used a polar representation $\sigma+i \vec{\tau} \cdot \vec{\pi} \gamma_{5} \equiv$ $\chi U_{5}$ for convenience [17. The first term in (4) with the coupling constant $g$ is a standard chiral invariant coupling in the linear $\sigma$ model. The second term with a new parameter $m_{0}$, which is usually not taken into account in the literature, is also chiral invariant and non-singular.

With the dynamical breaking of chiral symmetry $\left(\langle\sigma\rangle_{\text {vac }} \equiv \sigma_{0} \neq 0\right)$, eq.(田) expanded in terms of $\sigma / \sigma_{0}$ and $\vec{\pi} / \sigma_{0}$ reads, $\mathcal{L}_{I}(N, M)=-m_{N} \bar{N} N-\bar{N}\left(g_{\mathrm{s}} \tilde{\sigma}+i g_{\mathrm{p}} \vec{\tau}\right.$. $\left.\vec{\pi} \gamma_{5}\right) N+\frac{1}{2}\left(m_{0} / \sigma_{0}^{2}\right) \bar{N} \vec{\pi}^{2} N+0\left(\tilde{\sigma}^{l \geq 1} \times \pi^{n \geq 1}\right)$, where $\tilde{\sigma}=$ $\sigma-\sigma_{0}, m_{N} \equiv m_{0}+g \sigma_{0}, g_{\mathrm{s}} \equiv g$ and $g_{\mathrm{p}} \equiv g_{\mathrm{s}}+m_{0} / \sigma_{0}$. Because of $m_{0}$, the standard constraint $g_{s}=g_{p}$ can be relaxed without conflicting with chiral symmetry. Also, the term proportional to $m_{0} \pi^{2}$ appears to preserve chiral symmetry. $g_{\mathrm{p}}$ is constrained by the Goldberger-Treiman relation; $g_{\mathrm{p}}=m_{N} / \sigma_{0} \simeq m_{N} / f_{\pi}=g_{\pi N}=13.5$. On the other hand, $g_{\mathrm{s}}$ is independent of $g_{\mathrm{p}}$ and can be treated as a free parameter. With this freedom, one can circumvent the well-known problem that $g_{s}=g_{p}$ combined with eq.(3) does not reproduce the known nuclear matter properties in the mean-field level [18]. We remark that the dilated chiral model [19] can also avoid $g_{s}=g_{p}$ away from the "dilaton" limit, and has been applied to study the scalar meson in nuclear matter 20.

In the following, we treat the effect of the meson-loop as well as the baryon density as a perturbation to the vacuum quantities. Therefore, our loop-expansion is valid only at relatively low densities. The full self-consistent treatment of the problem requires systematic resummation of loops similar to what was developed at finite $T$ [10]. Let us first consider the chiral condensate in nuclear matter $\langle\sigma\rangle$ and parametrize it as

$$
\langle\sigma\rangle \equiv \sigma_{0} \Phi(\rho) .
$$

In the linear density approximation, $\Phi(\rho)=1-C \rho / \rho_{0}$ with $C=\left(g_{\mathrm{s}} / \sigma_{0} m_{\sigma}^{2}\right) \rho_{0}$. Instead of using $g_{\mathrm{s}}$, we use $\Phi$ as a basic parameter in the following analysis. The plausible value of $\Phi\left(\rho=\rho_{0}\right)$ is $0.7 \sim 0.9$ [2].
The one-loop corrections to the self-energy for $\sigma$ can be read off from the diagrams in Fig. $1: \Sigma_{\sigma}(\omega ; \rho)=\Sigma_{\text {vac }}^{A}$ $+\Sigma_{\text {vac }}^{B}+\Sigma_{M F}(\rho)+\Sigma_{p h}(\rho) . \quad \Sigma_{\text {vac }}^{A}\left(\Sigma_{\text {vac }}^{B}\right)$ corresponds to Fig.1A and Fig.1B respectively. Only the latter has $\omega$ dependence and the imaginary part. The explicit formula for $\Sigma_{\text {vac }}^{A+B}$ renormalized in the $\overline{M S}$ scheme is given in Appendix A of [10].

$\Sigma_{M F}(\rho)$ corresponds to the mean field correction in the nuclear matter (Fig.1C). $\Sigma_{p h}(\rho)$ is a correction from the nuclear particle-hole excitation. We take only the density dependent part in these diagrams and neglect the problematic vacuum-loops of the nucleon [18].

The leading term in the mean-field part is easily estimated as

$$
\Sigma_{M F}(\rho)=\lambda \sigma_{0}\left(\langle\sigma\rangle-\sigma_{0}\right)=-\lambda \sigma_{0}^{2}(1-\Phi(\rho)),
$$

Leading term in the particle-hole part (Fig.1D) in terms of $k_{F}=\left(3 \pi^{2} \rho / 2\right)^{1 / 3}$ reads $\Sigma_{p h}(\rho) \simeq \frac{2 g_{s}^{2}}{5 \pi^{2}} \frac{k_{F}^{5}}{M^{3}}$, which starts from $O\left(\rho^{5 / 3}\right)$ and is not more than a few $\%$ of $\Sigma_{M F}(\rho)$ at $\rho=\rho_{0}$. This is in contrast to the case of the pion, where both $\Sigma_{M F}(\rho)$ and $\Sigma_{p h}(\rho)$ are proportional to $\rho$ and cancel with each other due to chiral symmetry. Because of this cancellation, we can neglect the two-loop contribution related to the medium modification of the low-momentum pions near the $2 m_{\pi}$ threshold.

Up to this order, $\operatorname{Im} \Sigma_{\sigma}$ solely comes from $\operatorname{Im} \Sigma_{\text {vac }}^{B}$, since there is no Landau damping and scalar-vector mixing for the $\sigma$-meson at rest in nuclear matter:

$$
\operatorname{Im} \Sigma_{\sigma}(\omega ; \rho)=\operatorname{Im} \Sigma_{\text {vac }}^{B}=-\frac{\lambda^{2}}{96 \pi} \sigma_{0}^{2} \sqrt{1-\frac{4 m_{0 \pi}^{2}}{\omega^{2}}},
$$

for $2 m_{\pi} \leq \omega \leq 2 m_{\sigma}$.

Now, let us look at the spectral function defined in (11). As we have already discussed, the threshold peak is prominent when $\operatorname{Re} D_{\sigma}^{-1}=\omega^{2}-m_{\sigma}^{2}-\operatorname{Re} \Sigma_{\sigma}=0$. In the parametrization given in (5), this condition is rewritten as $\Phi\left(\rho_{c}\right)=0.74 \quad($ case $(\mathrm{I}))$, and $\Phi\left(\rho_{c}\right)=0.76 \quad$ (case (II)). The numbers in the right hand side are insensitive to the parameters in eq.(3) as far as the physical quantities in the vacuum such as $f_{\pi}$ and $m_{\pi}$ are fixed. In the linear density formula $\Phi(\rho)=1-C \rho / \rho_{0}$ with a reasonable value $C \simeq 0.2$, we obtain $\rho_{c} \simeq 1.25 \rho_{0}$ which is not far from the normal nuclear matter density. This implies that we could see the threshold enhancement in experiments with heavy nuclear targets.

The spectral functions together with $\operatorname{Re} D_{\sigma}^{-1}(\omega)$ for two cases (I) and (II) are shown in Fig.2 and in Fig.3, respectively. In both figures, the characteristic enhancement just above the $2 m_{\pi}$ threshold is seen for $\rho \simeq \rho_{c}$ 21].

We notice that the enhancement is caused by (a) partial restoration of chiral symmetry where $m_{\sigma}^{*}$ approaches toward $m_{\pi}$, and (b) the cusp structure of $\operatorname{Re} D_{\sigma}^{-1}(\omega)$ at $\omega=2 m_{\pi}$; see the lower panels of Fig.2 and Fig.3. Although the cusp is not prominent at zero density, it eventually hits the real axis at $\rho=\rho_{c}$ because $\operatorname{Re} D_{\sigma}^{-1}(\omega)$ 
increases associated with $m_{\sigma}^{*} \rightarrow 2 m_{\pi}$. This is a general phenomenon for systems where the internal symmetry is partially restored in the medium [22]. Another important observation is that, even at densities well below the point where $m_{\sigma}^{*}$ and $m_{\pi}$ are degenerate, one can expect the spectral enhancement near the $2 m_{\pi}$ threshold [23].

To confirm the threshold enhancement associated with the partial chiral restoration, measuring $2 \pi^{0}$ and $2 \gamma$ in experiments with hadron/photon beams off the heavy nuclear targets should be most appropriate. Measuring $\sigma \rightarrow 2 \pi^{0} \rightarrow 4 \gamma$ is experimentally feasible [12, and one can avoid the possible $I=J=1$ background from the $\rho$ meson inherent in the $\pi^{+} \pi^{-}$measurement. Measuring the direct electromagnetic decay $\sigma \rightarrow 2 \gamma$ is also important because of the small final state interactions. However, the branching ratio is small in this case $(\mathrm{Br}$ $\left.=\Gamma_{\sigma \rightarrow 2 \gamma} / \Gamma_{\sigma \rightarrow 2 \pi}=O\left(10^{-5}\right)[\mathbb{8}]\right)$, and one needs to fight with large background of photons mainly coming from $\pi^{0}$ 's. Nevertheless, if the enhancement is prominent and changes rapidly as the mass number of the target nucleus, there is a chance to find the signal. There is also a possibility that one can detect dilepton through the scalar-vector mixing in matter: $\sigma \rightarrow \gamma^{*} \rightarrow e^{+} e^{-}[13]$. In this case, the dileptons are produced only when $\sigma$ has a finite three momentum.

To enhance the production cross section for the critical fluctuation in the $I=J=0$ channel, $\left(\mathrm{d},{ }^{3} \mathrm{He}\right)$ reactions is useful. The incident kinetic energies of the deuteron in the laboratory system $E$ can be estimated to be $1.1 \mathrm{GeV}<E<10 \mathrm{GeV}$, to cover the spectral function in the range $2 m_{\pi}<\omega<750 \mathrm{MeV}$ [24].

Recently CHAOS collaboration 14 measured the $\pi^{+} \pi^{ \pm}$invariant mass distribution $M_{\pi^{+} \pi^{ \pm}}^{A}$ in the reaction $A\left(\pi^{+}, \pi^{+} \pi^{ \pm}\right) X$ with the mass number $A$ ranging from 2 to 208: They observed that the yield for $M_{\pi^{+} \pi^{-}}^{A}$ near the $2 m_{\pi}$ threshold is close to zero for $A=2$, but increases dramatically with increasing $A$. They identified that the $\pi^{+} \pi^{-}$pairs in this range of $M_{\pi^{+} \pi^{-}}^{A}$ is in the $I=J=0$ state. Attempts so far in hadronic models without considering the partial chiral restoration failed to reproduce this enhancement 25:26]. On the other hand, the invariant mass distribution presented in 14. near $2 m_{\pi}$ threshold for large $A$ has a close resemblance to our model calculation in Fig.2, which suggests that this experiment may already provide a hint about how the chiral symmetry is (partially) restored at finite density.

In summary, we have shown that the spectral function in the $I=J=0$ channel has a large enhancement near the $2 m_{\pi}$ threshold even at nuclear matter density due to the partial chiral restoration. Detection of the dipion and diphoton spectral distribution in the reactions of hadron/photon with heavy nucleus is suitable to confirm the idea of partial chiral restoration in nuclei.
This work was initiated when T. H. and T.K. attended GSI workshop on Hadrons in Matter (July 13, 1998). They are grateful to B. Friman, W. Nörenberg, and the theory department at GSI for their invitation and warm hospitality. T.K. thanks H. Pirner, C. Wetterich and the members of Institute for Theoretical Physics, Heidelberg University for their hospitality. He also thanks DAAD to make his stay in Germany possible.

Note added: After the completion of this paper, we got aware of a very recent study on the CHAOS data; R. Rapp et al., nucl-th/9810007. They do not address the question of the partial chiral restoration, and the underlying medium effect responsible for the near-threshold enhancement is different from ours.

[1] Y. Nambu and G. Jona-Lasinio, Phys. Rev. 122, 345 (1961).

[2] T. Hatsuda and T. Kunihiro, Phys. Rep. 247, 221 (1994). G. E. Brown and M. Rho, Phys. Rep. 269, 333 (1996). See also, B. Friman, nuc-th/9801053 (1998).

[3] See examples quoted in Basic Notion of Condensed Matter Physics, P. W. Anderson, (Benjamin, California, 1984); Phasenübergänge and Kritische Phänomene, W. Gebhardt and U. Krey, (Friedr. Vieweg \& Sohn, Wiesbaden, 1980).

[4] P. C. Hohenberg and B. I. Halperin, Rev. Mod. Phys. 49, 435 (1977). S. Sachdov, cond-mat/9705266.

[5] T. Alm, G. Röpke and M. Schmidt, Phys. Rev. C50, 31 (1994).

[6] T. Hatsuda and T. Kunihiro, Phys. Lett. 145(1984) 7; Phys. Rev. Lett. 55, 158 (1985); Phys. Lett. B185, 304 (1987).

[7] H. A. Weldon, Phys. Lett. B274, 133 (1992). C. Song and V. Koch, Phys. Lett. B404, 1 (1997).

[8] Particle Data Group, Eur. Phys. J. C 363, 390 (1998). See also the recent analyses of $\sigma:$ N. A. Törnqvist and M. Roos, Phys. Rev. Lett. 76, 1575 (1996); M. Harada, F. Sannino and J. Schechter, Phys. Rev. D54, 1991 (1996); S. Ishida et al., Prog. Theor. Phys. 98, 1005 (1997); J. A. Oller, E. Oset and J. R. Peláez, Phys. Rev. Lett. 80, 3452 (1998); K. Igi and K. Hikasa, Phys. Rev. D59, 034005 (1999).

[9] Most of the studies on the light $\sigma$-meson have been based on the reaction $\pi+N \rightarrow \pi \pi N$. Since $\Gamma_{\sigma \rightarrow 2 \gamma} \simeq 10 \mathrm{keV}$ [8], a feasible alternative way to study the properties of $\sigma$ in the vacuum is to employ the Primakoff production of $\sigma$ with a photon beam off the nuclear target. See, Letter of Intent by LEPS Collaboration for the laser electron photon project at Spring-8, Japan.

[10] S. Chiku and T. Hatsuda, Phys. Rev. D57, R6 (1998), ibid. D58, 076001 (1998).

[11] The measurement of $2 \pi^{0}$ through $4 \gamma$ detection was first suggested in 12]. The detection of $2 \gamma$ was suggested in the last reference of [6]. Other possibilities such as the 
lepton pair production through the vector-scalar mixing in nuclear medium has been discussed in 13.

[12] H. Shimizu, in Proceedings of $X V R C N P$ Osaka International Symposium, Nuclear Physics Frontiers with Electro-weak Probes (FRONTIER 96), (Osaka, March 78, 1996), p.161.

[13] T. Kunihiro, Prog. Theor. Phys. Supplement 120, 75 (1995), in which standard nuclear experiments with the beams of proton, light ions, pion and photon are proposed to produce the $\sigma$ in nuclei for studing partial chiral restoration.

[14] F. Bonutti et al. (CHAOS Collaboration), Phys. Rev. Lett. 77, 603 (1996).

[15] J. Gasser and H. Leutwyler, Ann. Phys. 158, 142 (1984).

[16] B. W. Lee, Nucl. Phys. B9, 649 (1969). B. W. Lee, Chiral Dynamics, (Gordon and Breach, New York, 1972). L. H. Chan and R. W. Haymaker, Phys. Rev. D7, 402 (1973); ibid. D10, 4170 (1974).

[17] The leading correction to eq.(44) from the explicit symmetry breaking can be absorbed into the couplings $g, m_{0}$ as far as the $\sigma$-nucleon coupling is concerned.

[18] B. D. Serot and J. D. Walecka, Int. J. Mod. Phys. E6, 515 (1997).

[19] S. R. Beane and U. van Kolck, Phys. Lett. B328, 137 (1994).

[20] Y. Kim and H. K. Lee, Phys. Rev. C55, 3100 (1997). See also, Y. Kim, H. K. Lee and M. Rho, Phys. Rev. C52, 1184 (1995).

[21] In the one-loop level, the spectral function satisfies the sum rule $\int_{0}^{\infty} \rho_{\sigma}(\omega) d \omega^{2}=1$ [10].

[22] For $T>T_{c}$, one can make model independent statement on the critical enhancement on the basis of the QCD operator product expansion: See, e.g. S. Huang and M. Lissia, Phys. Rev. D52, 1134 (1995).

[23] The nuclear force mediated by $\sigma$ is related to the spectral function by the Laplace transform. Therefore, a large spectral shift is smeared by integration. Nevertheless, the strength and the range of the potential has some changes. See, R. Rapp, R. Machleidt, J. W. Durso and G. E. Brown, nucl-th/9706006 on this point.

[24] This estimate of the kinetic energy is in accordance with those for stopped $\eta$ meson production; R.S. Hayano, S. Hirenzaki and A. Gillitzer, nucl-th/9806012.

[25] Z. Aouissat, R. Rapp, G. Chanfray, P. Schuck and J. Wambach, Nucl. Phys. A581, 471 (1995). R. Rapp, J. W. Durso and J. Wambach, Nucl. Phys. A596, 436 (1996) and references therein.

[26] H. C. Chiang, E. Oset, and M. J. Vicente-Vacas, Nucl. Phys. A644, 77 (1998).

\begin{tabular}{|c|c|ccc|}
\hline \hline & $m_{\sigma}^{\text {peak }}(\mathrm{MeV})$ & $\sqrt{-\mu^{2}}(\mathrm{MeV})$ & $\lambda / 4 \pi$ & $h^{1 / 3}(\mathrm{MeV})$ \\
\hline (I) & 550 & 284 & 5.81 & 123 \\
(II) & 750 & 375 & 9.71 & 124 \\
\hline \hline
\end{tabular}

TABLE I. Parameters for $m_{\sigma}^{\text {peak }}=550,750 \mathrm{MeV}$

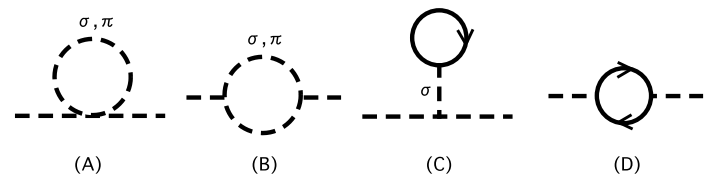

FIG. 1. One-loop self energy. The dashed line denotes either $\sigma$ or $\pi$. The solid line denotes the nucleon.
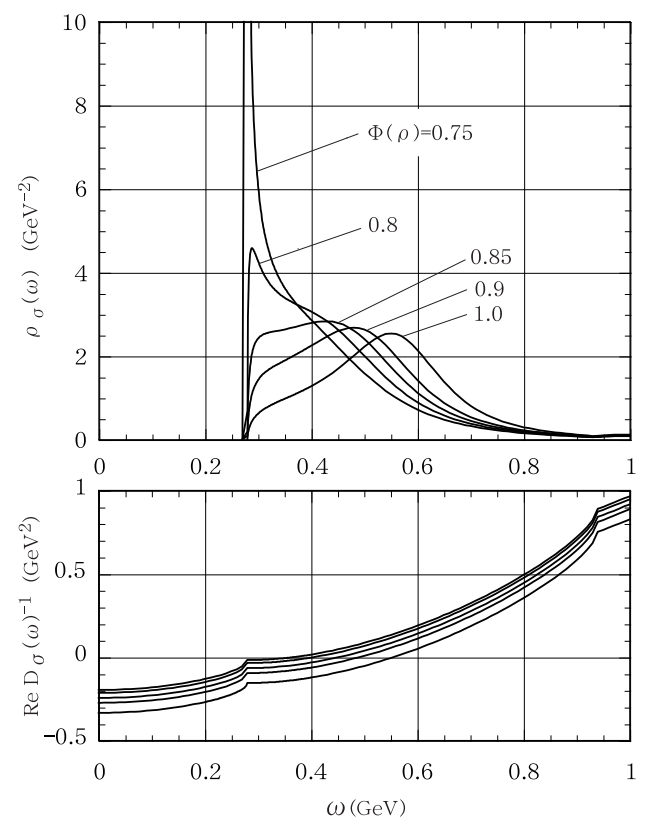

FIG. 2. Spectral function for $\sigma$ and the real part of the inverse propagator for several values of $\Phi=\langle\sigma\rangle / \sigma_{0}$ with $m_{\sigma}^{\text {peak }}=550 \mathrm{MeV}$ (case (I) in TABLE I). In the lower panel, $\Phi$ decreases from bottom to top.
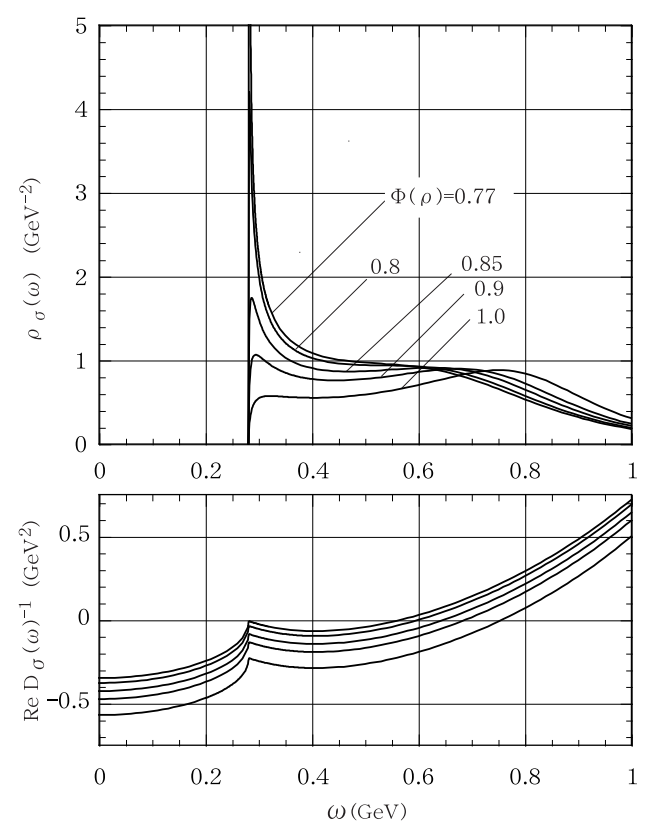

FIG. 3. Same with Fig.2 for $m_{\sigma}^{\text {peak }}=750 \mathrm{MeV}$ (case (II) in TABLE I. 